\title{
Evaluation of FISH for Blood Cultures under Diagnostic Real-Life Conditions
}

\author{
Annalena Reitz ${ }^{1}$, Sven Poppert ${ }^{2,3}$, Melanie Rieker ${ }^{4}$ and Hagen Frickmann ${ }^{5,6^{*}}$ \\ ${ }^{1}$ University Hospital of the Goethe University, Frankfurt/Main, Germany \\ ${ }^{2}$ Swiss Tropical and Public Health Institute, Basel, Switzerland \\ ${ }^{3}$ Faculty of Medicine, University Basel, Basel, Switzerland \\ ${ }^{4}$ MVZ Humangenetik Ulm, Ulm, Germany \\ ${ }^{5}$ Department of Microbiology and Hospital Hygiene, Bundeswehr Hospital Hamburg, Hamburg, Germany \\ ${ }^{6}$ Institute for Medical Microbiology, Virology and Hygiene, University Hospital Rostock, Rostock, Germany
}

Received: 04 September 2018; accepted: 18 September 2018

\begin{abstract}
Background: The study assessed a spectrum of previously published in-house fluorescence in-situ hybridization (FISH) probes in a combined approach regarding their diagnostic performance with incubated blood culture materials.

Methods: Within a two-year interval, positive blood culture materials were assessed with Gram and FISH staining. Previously described and new FISH probes were combined to panels for Gram-positive cocci in grape-like clusters and in chains, as well as for Gram-negative rod-shaped bacteria. Covered pathogens comprised Staphylococcus spp., such as S. aureus, Micrococcus spp., Enterococcus spp., including E. faecium, E. faecalis, and E. gallinarum, Streptococcus spp., like S. pyogenes, S. agalactiae, and S. pneumoniae, Enterobacteriaceae, such as Escherichia coli, Klebsiella pneumoniae and Salmonella spp., Pseudomonas aeruginosa, Stenotrophomonas maltophilia, and Bacteroides spp.

Results: A total of 955 blood culture materials were assessed with FISH. In 21 (2.2\%) instances, FISH reaction led to non-interpretable results. With few exemptions, the tested FISH probes showed acceptable test characteristics even in the routine setting, with a sensitivity ranging from $28.6 \%$ (Bacteroides spp.) to $100 \%$ (6 probes) and a specificity of $>95 \%$ in all instances.

Conclusion: If sophisticated rapid diagnostic methods like mass spectrometry from blood culture materials are not available, FISH provides an option for rapid differentiation for laboratories in resource-limited settings.
\end{abstract}

Keywords: fluorescence in-situ hybridization, blood culture, rapid diagnostics, molecular diagnostics, sepsis

\section{Introduction}

Fluorescence in-situ hybridization (FISH) is a diagnostic technique which allows the identification of pathogens on species or genus level by the binding of short fluorescence-tagged pathogen-specific DNA probes to ribosomal RNA of microorganisms. Subsequently, analysis is performed under a fluorescence microscope. Use of multi-well slides allows the application of whole panels of FISH probes with the same specimen in a parallel approach [1]. This procedure, the so-called multiprobe concept, allows the exclusion of auto-fluorescence and non-specific binding by assessing fluorescence intensity of probes, which should not lead to positive results. If positive signals are provided by different FISH probes leading to contradicting results, the overall result is "non-interpretable" but at least not "false positive" in this way.

Nearly 20 years ago, FISH was identified as an easy and rapid procedure to provide additional information in addition to Gram staining from positive blood culture materials. As early as at the beginning of the new millennium, small and in part poorly evaluated probe panels for the identification of blood culture pathogens were published [2], but insufficient standardization limited the implementation of the technique for the diagnostic routine setting [3].

\footnotetext{
* Author for correspondence: Department of Microbiology and Hospital Hygiene, Bundeswehr Hospital Hamburg, Bernhard Nocht Str. 74, 20359 Hamburg, Germany; E-mail: Frickmann@bni-hamburg.de; Phone: 0049-406947-28700; Fax: 0049-40-6947-28709.
}

Due to the experience that various previously published probes did not show acceptable performance characteristics in our hands, we started to develop new probes and to publish them individually [4-7]. Accordingly, some of the data presented here have partially been published in a pathogen-specific way. Now, the entire data-set is reevaluated in a concluding synopsis.

Due to the continuous evaluation process, the data are incoherent. This means that the probe sets were adjusted in the course of the study, if binding characteristics of individual probes were considered as inappropriate. In the here described assessment, only the results for the probes and screening algorithms, which were proven to be useful in our hands and could thus be used in the course of the whole evaluation, were described. Due to the stepwise, Gram-staining-dependent, species-specific evaluation approaches, the here described FISH assessments strongly overlap with previous reports [4-7]. However, the samples are not identical, and therefore, the results are not equal.

In the meantime, MALDI-TOF-MS (matrix assisted laser desorption ionization-time of flight-mass spectrometry) is the predominantly applied method for the early identification of pathogen species from incubated blood culture materials $[8,9]$. Nevertheless, cost-efficient FISH could be an option for resource-limited settings, where expensive MALDI-TOF-MS equipment is not affordable, e.g., in non-industrialized tropical areas $[1,7]$. However, this requires availability of a fluorescence microscope and of well-trained laboratory personnel, so manpower can compensate a lack of sophisticated technology.

This is an open-access article distributed under the terms of the Creative Commons Attribution-NonCommercial 4.0 International License (https://creativecommons.org/licenses/by-nc/4.0/), which permits unrestricted use, distribution, and reproduction in any medium for non-commercial purposes, provided the original author and source are credited, a link to the CC License is provided, and changes - if any - are indicated. 
In the here described study, the diagnostic performance of predominantly published FISH probes for blood culture diagnostics was assessed during a two-year interval under real-life-like conditions in a diagnostic routine laboratory of a German university hospital.

\section{Materials and Methods}

Study Setting. During a two-year interval, positive blood cultures were assessed by FISH in addition to Gram staining, as soon as they were detected positive by automated blood culture. The FISH results were compared with the definite diagnostic results after cultural growth and biochemical identification via VITEK or API (bioMérieux, Marcy-l'Étoile, France). The study was performed at the Institute for Medical Microbiology of the University Hospital of Ulm.

Inclusion and Exclusion Criteria. Positive blood culture materials were included in the FISH assessment if they showed either Gram-positive cocci in grape-like clusters or Gram-positive cocci in doubles or chains or Gram-negative rod-shaped bacteria. Positive blood culture materials with other morphology of microorganisms in Gram-stain and negative blood culture materials were excluded. There was no exclusion of copy strains, and both aerobic and anaerobic cultures from the same patients were considered as distinct samples. However, materials were excluded from further assessment if they led to non-interpretable FISH results due to multiple positive FISH signals in spite of only one type of observed morphology in Gram-stain as it may occur due to autofluorescence phenomena or in the case of failed reactions of the internal control FISH. In such instances, FISH cannot be interpreted [1]. Applying these exclusion criteria, 21 samples had to be excluded as summarized in Table 1.

FISH Procedure. Depending on the results of Gramstaining of positively tested blood culture materials, different panels of FISH probes were applied, as shown in Table 2. Specific probes were labelled with the red fluorescence dye Cy3 (cyanine), while the pan-eubacterial probe, which was used as the internal reaction control, was labelled with the green fluorescence dye FAM (carboxyfluorescein). Competitor probes are non-labelled probes which are added to block nonspecific probe binding to phylogenetically closely related organisms. For enterococci and Salmonella spp., 2 distinct FISH probes and, in the latter case, also 2 distinct probecompetitor-probe-combinations were available (Table 2).

FISH was performed as described [1]. In short, slides with blood culture material were fixed with $100 \%$ methanol and

Table 1. Excluded strains due to non-interpretable results, i.e., multiple positive signals in the multi-probe approach or failed reactions in the internal control FISH

Acinetobacter lwoffii $(n=1)$
Brevundimonas diminuta $(n=1)$
Comamonas acidovorans $(n=1)$
Enterococcus faecium $(n=1)$
Escherichia coli $(n=1)$
Flavimonas oryzihabitans $(n=1)$
Gemella haemolysans $(n=2)$
Koccuria spp. $(n=1)$
Micrococcus lylae $(n=1)$
Moraxella catarralis $(n=2)$
Moraxella osloensis $(n=1)$
not further differentiated Gemella $\mathrm{spp} .(n=1)$
not further differentiated
Gram-negative rod-shaped bacteria $(n=2)$
not further differentiated Pseudomonas $\mathrm{sp} .(n=1)$
Pseudomonas aeruginosa $(n=1)$
Staphylococcus epidermidis $(n=1)$
Stomatococus mucilaginosus $(n=1)$
Streptococcus mitis $(n=1)$

air-dried. If Gram-positive cocci were seen, permeabilization was performed for $5 \mathrm{~min}$ with a lysis buffer containing $1 \mathrm{mg} /$ $\mathrm{mL}$ lysozyme and $2 \mu \mathrm{g} / \mathrm{mL}$ lysostaphine in $10 \mathrm{mM}$ Tris $\mathrm{HCl}$ at $46^{\circ} \mathrm{C}$. No lysis was necessary for Gram-negative organisms. All probes were designed to work with $30 \%$ formamide in the hybridization buffer containing $0.9 \mathrm{M} \mathrm{NaCl}, 20 \mathrm{mM}$ Tris $\mathrm{HCl}$, and $0.01 \%$ sodium dodecyl sulphate (SDS). Hybridization time was $60 \mathrm{~min}$, followed by washing for about 15 min with a washing buffer containing $0.102 \mathrm{M} \mathrm{NaCl}, 5 \mathrm{mM}$ EDTA, $20 \mathrm{mM}$ Tris $\mathrm{HCl}$, and $0.01 \%$ SDS. Prior to assessment on a fluorescence microscope, counterstaining of the bacterial DNA was performed with 4',6-diamidin-2-phenylindol (DAPI).

In Silico Evaluation of the FISH Probes. In silico evaluation of all tested FISH probes was performed using the software probecheck (http://131.130.66.200/cgi-bin/probecheck/ content.pl?id=home). The results are shown in Supplementary material 1.

Statistical Assessment. Descriptive assessment was performed to calculate sensitivity, specificity, positive predictive value, and negative predictive value using the software Microsoft Excel ${ }^{\circledR}$.

Ethical Standards. Not applicable because no patient data or primary sample materials were used.

\section{Results}

Assessed Blood Culture Materials and Identification Results by the Reference Methods. Altogether, a total of 733 pathogens from positive aerobic blood culture materials and 222 pathogens from positive anaerobic blood culture materials were included in the study during the two-year-assessment period. They comprised 33 materials with mixed bacterial cultures comprising 29 materials with double infections and 4 materials with triple infections.

Altogether, 955 Gram-positive cocci in grape-like clusters, doubles, or chains, as well as Gram-negative, rod-shaped bacteria were identified. As stated above, 21 out of 955 (2.2\%) samples had to be excluded, applying the exclusion criteria. These excluded strains comprised Acinetobacter lwoffii $(n=1)$, Brevundimonas diminuta $(n=1)$, Comamonas acidovorans $(n=1)$, Enterococcus faecium $(n=1)$, Escherichia coli $(n=1)$, Flavimonas oryzihabitans $(n=1)$, Gemella haemolysans $(n=2)$, Kocuria spp. $(n=1)$, Micrococcus lylae $(n=1)$, Moraxella catarrhalis $(n=2)$, M. osloensis $(n=1)$, not further differentiated Gemella spp. $(n=1)$, not further differentiated Gramnegative rod-shaped bacteria $(n=2)$, not further differentiated Pseudomonas spp. $(n=1)$, P. aeruginosa $(n=1)$, Staphylococcus epidermidis $(n=1)$, Stomatococcus mucilaginosus $(n=1)$, and Streptococcus mitis $(n=1)$. The remaining 934 pathogens that were included into further assessment comprised 534 Grampositive cocci in grape-like clusters, i.e., Staphylococcus aureus $(n=84), S$. auricularis $(n=1), S$. capitis $(n=1), S$. caprae $(n=1), S$. cohnii $(n=1), S$. epidermidis $(n=356), S$. haemolyticus $(n=10), S$. hominis $(n=9), S$. saccharolyticus $(n=1)$, $S$. simulans $(n=3)$, Micrococcus luteus $(n=10)$, and not further differentiated coagulase negative staphylococci $(n=57)$. Further, 148 Gram-positive cocci in doubles or chains were observed, comprising Enterococcus faecalis $(n=38), E$. faecium $(n=41)$, E. gallinarum $(n=3)$, Lactococcus lactis $(n=3)$, not further differentiated alpha-hemolytic Streptococcus spp. $(\mathrm{n}=2), S$. agalactiae $(\mathrm{n}=5), S$. anginosus $(n=6)$, $S$. dysgalactiae $(n=3), S$. dysgalactiae subsp. equisimilis $(n=1), S$. mitis $(n=18), S$. oralis $(n=4), S$. pneumoniae $(n=16), S$. pyogenes $(n=3)$, S. salivarius $(n=1)$, and $S$. sanguis $(n=4)$. Further, 252 Gram-negative rod-shaped bacteria were identified as Acinetobacter baumannii $(n=1)$, A. lwoffii $(n=2)$, Bacteroides fragilis $(n=4), B$. thetaiotaomicron $(n=1)$, Citrobacter freundii $(n=4), C$. koseri $(n=2)$, Enterobacter 
Table 2. Applied FISH probe panels for blood culture diagnostics

\begin{tabular}{|c|c|c|}
\hline Target organism & Probe sequence & Reference \\
\hline \multicolumn{3}{|c|}{ Pan-eubacterial probe combined with all panels } \\
\hline All bacteria & 5'-GCT-GCC-TCC-CGT-TAG-GAG-T-3“ & [27] \\
\hline \multicolumn{3}{|c|}{ Panel for Gram-positive cocci in grape-like clusters } \\
\hline Staphylococcus aureus & 5'-GAA-GCA-AGC-TTC-TCG-TCC-G-3' & [3] \\
\hline Staphylococcus spp. & 5'-TCC-TCC-ATA-TCT-CTG-CGC-3' & [3] \\
\hline Micrococcus spp. & 5'-GTA-TCT-CTA-CGG-CGA-TCG-3' & This study \\
\hline \multicolumn{3}{|c|}{ Panel for Gram-positive cocci in doubles or chains } \\
\hline Enterococcus faecalis & 5'-GAA-AGC-GCC-TTT-CAC-TCT-TAT-GC-3' & [4] \\
\hline Enterococcus faecium & 5'-TTC-ACA-CAA-TCG-TAA-CAT-CCT-A-3' & [4] \\
\hline Enterococcus gallinarum & 5'-ATT-CAC-AAC-TGT-GTA-ACA-TCC-TAT-3' & [4] \\
\hline Enterococcus spp. & 5'-CAC-CGC-GGG-TCC-ATC-CAT-CA-3' and 5'-CAG-TTC-TCT-GCG-TCT-ACC-TC-3' & [4] \\
\hline Streptococcus agalactiae & 5'-GTA-AAC-ACC-AAA-CMT-CAG-CG-3' & [28] \\
\hline Streptococcus pneumoniae & $\begin{array}{l}\text { 5'-GTG-ATG-CAA-GTG-CAC-CTT-3' in combination with the competitor probe } \\
\text { 5'-GTG-ATG-CAA-TTG-CAC-CTT-3' }\end{array}$ & [3] \\
\hline Streptococcus pyogenes & 5'-CTA-ACA-TGC-GTT-AGT-CTC-TC-3' & This study \\
\hline \multirow{2}{*}{\multicolumn{3}{|c|}{ Panel for Gram-negative rod-shaped bacteria }} \\
\hline & & \\
\hline Acinetobacter spp. & 5'-TTA-GGC-CAG-ATG-GCT-GCC-3' & [6] \\
\hline Bacteroides spp. & $\begin{array}{l}\text { 5'-CAT-CCT-TCA-CGC-TAC-TTG-GCT-GG-3' in combination with the two competitor probes } \\
\text { 5'-TCC-TTC-ACG-CGA-CTT-GGC-TGG-TT-3' and 5'-TCC-TGC-ACG-CTA-CTT-GGC-TGG-T-3' }\end{array}$ & This study \\
\hline Bacteroides spp. / Prevotella spp. & $\begin{array}{l}\text { 5'-CAT-CCT-TCA-CGC-TAC-TTG-GCT-GG-3' in combination with the competitor probe } \\
\text { 5'-TCC-TTC-ACG-CGA-CTT-GGC-TGG-TT-3' }\end{array}$ & This study \\
\hline Enterobacteriaceae & 5'-CCC-CCW-CTT-TGG-TCT-TGC-3' & [3] \\
\hline Escherichia coli & 5'-GCG-GGT-AAC-GTC-AAT-GAG-CAA-AGG-3' & This study \\
\hline Klebsiella pneumoniae & 5'-CCT-ACA-CAC-CAG-CGT-GCC-3' & [3] \\
\hline Pseudomonas aeruginosa & 5'-CCA-CTT-TCT-CCC-TCA-GGA-CG-3' & This study \\
\hline \multirow{2}{*}{ Salmonella spp. } & 5'-TGC-GCT-TTT-GTG-TAC-GGG-GCT-3' in combination with the competitor probe & [7] \\
\hline & $\begin{array}{l}\text { 5'-GTG-CAT-TTT-TGT-GTA-CGG-GGC-3' and 5'-CTT-CAC-CTA-CGT-GTC-AGC-G-3' } \\
\text { in combination with the competitor probe 5'-TCA-CCT-ACA-TAT-CAG-CGT-GC-3' }\end{array}$ & \\
\hline Stenotrophomonas maltophilia & 5'-GTC-GTC-CAG-TAT-CCA-CTG-C-3' & [26] \\
\hline
\end{tabular}

aerogenes (Klebsiella aerogenes) $(n=8)$, E. amnigenus $(n=1)$, E. cloacae $(n=12)$, E. hormaechei $(n=1)$, Escherichia coli $(n=126)$, Klebsiella oxytoca $(n=7), K$. pneumoniae $(n=27)$, Morganella morganii $(n=1)$, not further differentiated Acinetobacter spp. $(n=6)$, not further differentiated Bacteroides spp. $(n=2)$, not further differentiated Citrobacter spp. $(n=1)$, not further differentiated Pantoea spp. $(n=1), P$. agglomerans $(n=1)$, Pseudomonas aeruginosa $(n=30)$, Salmonella ser Bredeney $(n=1), S$. ser Enteritidis $(n=3), S$. ser Typhi $(n=2)$, Serratia liquefaciens $(n=1), S$. marcescens $(n=4)$, and Stenotrophomonas maltophilia $(n=3)$ (Table 3$)$.

In-silico Evaluation and Diagnostic Performance of the Assessed FISH Probes. Details of the in-silico evaluation of the assessed FISH probes are presented in the supplementary material (Supplementary material 1) and summarized in Table 4. As indicated in Table 4, potential cross-binding was considered as likely in the case of mismatches up to 2 bases. In-silico matching with the target organisms was $26.9-100 \%$ for the 0 -mismatch range, $0-100 \%$ for the 1-base-mismatch range and $0-99.9 \%$ for the 2-bases-mismatch range (Table 4). Of note, even probes that showed good binding characteristics in the later in-vitro evaluation like the probe for $S$. aureus (Table 5) were associated with only moderate matching with deposited sequences in the in-silico evaluation (Table 4).

The in-vitro performance characteristics of the assessed FISH probes are detailed in Table 5. While the specificity of all described FISH approaches was better than $95 \%$, sensitivity ranged from $28.6 \%$ for Bacteroides spp. to $100 \%$ for Micrococcus spp., E. gallinarum, S. pyogenes, Acinetobacter spp., Klebsiella pneumoniae, and Salmonella spp., respectively. Low percentage values, however, were influenced by disproportional high effects of single missed bindings in the case of species which were very rarely identified, like $S$. agalactiae (Table 5).

The details on lacking or incorrect binding of FISH probes are provided in Table 6 . In detail, the $S$. aureus probe failed to bind to 3 S. aureus isolates. Of note, the Staphylococcus spp. probe was once erroneously used with Gram-positive cocci in chains and showed cross-binding with a $S$. mitis isolate. This erroneous use is not shown in Table 5. The probe altogether failed in the cases of $2 S$. aureus, $3 \mathrm{~S}$. epidermidis, and 4 not further identified Staphylococcus spp. The E. faecalis probe missed 2 E. faecalis isolates, and the E. faecium probe missed 8 E. faecium isolates. In contrast, the E. gallinarum probe showed cross-binding with 2 E. faecium isolates. The Enterococcus spp. probes failed to identify 3 Enterococcus spp. strains, i.e., 1 E. faecalis and 2 E. faecium. The $S$. agalactiae probe missed $1 S$. agalactiae isolate, and the S. pneumoniae probe missed $2 S$. pneumoniae isolates. The $S$. pyogenes probe showed cross-binding with $1 S$. anginosus strain. The Streptococcus sp. probe missed $1 \mathrm{~S}$. mitis but identified all 3 Lactococcus lactis isolates. Therefore, it has to be considered as a Streptococcus spp./Lactococcus spp. probe. The Bacteroides spp. probe missed 5 out of 7 Bacteroides spp., i.e., 3 Bacteroides fragilis, 1 Bacteroides thetaiotaomicron, and 1 not further identified Bacteroides sp. strain if used with both competitor probes against Prevotella spp. and Porphyromonas spp. If it was used as a Bacteroides spp./Prevotella spp. probe in conjunction with only the competitor probe against Porphyromonas spp., 3 out of 7 Bacteroides spp., i.e., 1 Bacteroides fragilis and 2 not further identified Bacteroides spp. were missed. The Enterobacteriaceae probe failed in 4 instances with 4 E. coli. The Escherichia coli probe missed 5 E. coli and showed cross-binding with 1 Klebsiella oxytoca isolate. The Klebsiella pneumoniae probe showed cross-binding with 1 E. amnigenus strain, 2 Klebsiella oxytoca strains, and 1 Serratia marcescens. The $P$. aeruginosa probe missed $3 P$. aeruginosa isolates, and the $S$. maltophilia probe missed 1 out of 3 S. maltophilia in blood culture.

\section{Discussion}

The application of FISH for rapid blood culture diagnostics is not a new innovation. In parallel to MALDI-TOF-MS for rapid blood culture diagnostics, FISH was evaluated in various commercial and non-commercial approaches for this purpose. 
Table 3. Distribution of species in samples which were included in the assessment

\begin{tabular}{|c|c|c|}
\hline Groups & Species & $\begin{array}{c}\text { Numbers of } \\
\text { species }\end{array}$ \\
\hline $\begin{array}{l}534 \text { Gram-positive } \\
\text { cocci in grape-like } \\
\text { clusters }\end{array}$ & $\begin{array}{c}\text { Staphylococcus aureus } \\
\text { Staphylococcus auricularis } \\
\text { Staphylococcus capitis } \\
\text { Staphylococcus caprae } \\
\text { Staphylococcus cohnii } \\
\text { Staphylococcus epidermidis } \\
\text { Staphylococcus haemolyticus } \\
\text { Staphylococcus hominis } \\
\text { Staphylococcus saccharolyticus } \\
\text { Staphylococcus simulans } \\
\text { Micrococcus luteus } \\
\text { not further differentiated } \\
\text { coagulase negative staphylococci }\end{array}$ & $\begin{array}{c}84 \\
1 \\
1 \\
1 \\
1 \\
356 \\
10 \\
9 \\
1 \\
3 \\
10 \\
57\end{array}$ \\
\hline $\begin{array}{l}148 \text { Gram-positive } \\
\text { cocci in doubles } \\
\text { or chains }\end{array}$ & $\begin{array}{c}\text { Enterococcus faecalis } \\
\text { Enterococcus faecium } \\
\text { Enterococcus gallinarum } \\
\text { Lactococcus lactis } \\
\text { not further differentiated alpha- } \\
\text { hemolytic Streptococcus spp. } \\
\text { Streptococcus agalactiae } \\
\text { Streptococcus anginosus } \\
\text { Streptococcus dysgalactiae } \\
\text { Streptococcus dysgalactiae } \\
\text { subsp. equisimilis } \\
\text { Streptococcus mitis } \\
\text { Streptococcus oralis } \\
\text { Streptococcus pneumoniae } \\
\text { Streptococcus pyogenes } \\
\text { Streptococcus salivarius } \\
\text { Streptococcus sanguis }\end{array}$ & $\begin{array}{c}38 \\
41 \\
3 \\
3 \\
2 \\
\\
5 \\
6 \\
3 \\
1 \\
\\
18 \\
4 \\
16 \\
3 \\
1 \\
4\end{array}$ \\
\hline $\begin{array}{l}252 \text { Gram-negative } \\
\text { rod-shaped bacteria }\end{array}$ & $\begin{array}{c}\text { Acinetobacter baumannii } \\
\text { Acinetobacter lwoffii } \\
\text { Bacteroides fragilis } \\
\text { Bacteroides thetaiotaomicron } \\
\text { Enterobacter aerogenes } \\
\text { Enterobacter amnigenus } \\
\text { Enterobacter cloacae } \\
\text { Citrobacter freundii } \\
\text { Enterobacter hormaechei } \\
\text { Escherichia coli } \\
\text { Citrobacter koseri } \\
\text { Klebsiella pneumoniae } \\
\text { Klebsiella oxytoca } \\
\text { Morganella morganii } \\
\text { not further differentiated } \\
\text { Acinetobacter spp. } \\
\text { not further differentiated } \\
\text { Bacteroides spp. } \\
\text { not further differentiated } \\
\text { Citrobacter } \text { spp. } \\
\text { not further differentiated } \\
\text { Pantoea } \text { spp. } \\
\text { Pantoea agglomerans } \\
\text { Pseudomonas aeruginosa } \\
\text { Salmonella } \text { ser Bredeney } \\
\text { Salmonella } \text { ser Enteritidis } \\
\text { Salmonella } \text { ser Typhi } \\
\text { Serratia liquefaciens } \\
\text { Serratia marcescens } \\
\text { Stenotrophomonas maltophilia }\end{array}$ & $\begin{array}{c}1 \\
2 \\
4 \\
1 \\
8 \\
1 \\
12 \\
4 \\
1 \\
126 \\
2 \\
27 \\
7 \\
1 \\
6 \\
\\
2 \\
1 \\
\\
1 \\
1 \\
30 \\
1 \\
3 \\
2 \\
1 \\
4 \\
3\end{array}$ \\
\hline
\end{tabular}

Commercial applications comprise the use of patent-protected peptide-nucleic-acid (PNA)-FISH probes as developed and distributed by AdvanDx, Inc. (Vedbæk, Denmark) for the identification of pathogens in incubated blood culture materials. Respective PNA FISH approaches for the rapid diagnosis of blood culture pathogens comprised agents like Acinetobacter spp., C. albicans, E. coli, K. pneumonia, P. aeruginosa, and S. aureus [10-14].

Molecular beacon DNA probes for FISH-based blood culture diagnostics in a commercial hemo-FISH kit (miacom diagnostics, Düsseldorf, Germany) for both Gram-positive and Gram-negative pathogens have been introduced as well [15], next to various in-house approaches based on DNA FISH probes [5-7, 16-20].

For the presented study, in-silico evaluation of the probes was repeated with the updated database. The discrepancy, as exemplarily described for $S$. aureus in the Results section, between the observed in-vitro performance of the probes and the relatively poor in-silico matching is known from previous assessments. Even the current databases are not perfect, and older entries with wrong identifications are still included. Accordingly, in-vitro evaluations cannot be avoided.

This study assessed various published and few unpublished DNA FISH probes in a real-life-like observation at a German university hospital for their suitability for diagnostic FISH from blood culture materials. As detailed in the results, sensitivity and specificity in this real-life-like approach were slightly lower than suggested by previous evaluations under study conditions [16-0] including our own previous species-specific assessments [4-7]. Under the study conditions, cultured bacteria were directly tested by FISH in all contradictory cases, and in some cases, FISH was right in the end. In the here described assessment, such test repetition was not included, and the results were presented as obtained under routine-like conditions. Although phenotypical differentiation was used as reference standard for this assessment, it cannot be excluded that FISH results were nevertheless correct in some discrepant cases, as phenotypical methods can infrequently lead to incorrect results as well [21].

Anyway, very poor performance characteristics with less than $80-90 \%$ matching were only observed, like in the case of sensitivity of the $S$. maltophilia probe, if only very few samples were part of the assessment. Individual false reactions had a lot of impact under these circumstances, an undeniable limitation of the presented single-center assessment. As impressively shown for the example of the Bacteroides spp. probe, increases in specificity due to the adding of several competitor probes [1] can lead to reduction of sensitivity to unacceptably low values. Further, there have been various changes in the taxonomy of anaerobic bacteria in the recent years, and the applied biochemical identification procedures were shown to be associated with limited reliability as well [22].

The assessment of the usefulness of FISH probes requires the consideration of various factors. For example, FISH-based differentiation of staphylococci was confirmed to show good performance characteristics under routine-like conditions. Only a small probe panel was necessary for the discrimination of Gram-positive cocci in grape-like clusters, and FISH allowed a clearly visible microscopic distinction between coagulase-negative staphylococci and the clinically much more relevant species, Staphylococcus aureus. Successful commercial applications have been described [23].

For microorganisms with other morphology in Gram stain, like yeasts, which were not in the scope of this assessment but detailed elsewhere [24-25], Gram-positive cocci in doubles or chains, or even Gram-negative rod-shaped bacteria, considerably larger probe panels are required for differentiation. Especially for Gram-negative bacteria, FISH-based differentiation without resistance data is also less likely to allow clear-cut therapeutic decisions just based on the species identity. For anaerobic bacteria, FISH-based identification using the described approach currently makes little sense due to poor performance and debatable therapeutic consequences. Anyway, identification of anaerobic bacteria is difficult with conventional phenotypic methods as well [22], potentially resulting in many incorrect entries in the databases. Next to this, nomenclature of these species is still in progress. If this 
Table 4. In-silico matching of the assessed probes with target sequences in the 0 -mismatch range ( $0 \mathrm{MM})$, the 1-base-mismatch range (1 MM), and the 2-bases-mismatch range (2 MM) (-, not matches indicated)

\begin{tabular}{|c|c|c|c|c|}
\hline Probe $^{a}$ & Target organism & $0 \mathrm{MM}$ & $1 \mathrm{MM}$ & $2 \mathrm{MM}$ \\
\hline \multirow[t]{2}{*}{ ACS 16S 729} & \multirow[t]{2}{*}{ Acinetobacter spp. } & $61.7 \%$ & $57.6 \%$ & $9.7 \%$ \\
\hline & & $3114 / 5046$ & $276 / 479$ & $45 / 463$ \\
\hline $\begin{array}{l}\text { Entero all (variant } 1 \text { : "A" at the } \\
\text { wobble position) }\end{array}$ & Enterobacterales & $58.1 \%$ & $47.0 \%$ & $51.0 \%$ \\
\hline \multirow{2}{*}{$\begin{array}{l}\text { Entero all (variant } 2: \text { "T" at the } \\
\text { wobble position) }\end{array}$} & \multirow[t]{2}{*}{ Enterobacterales } & $46.5 \%$ & $55.7 \%$ & $54.0 \%$ \\
\hline & & $6526 / 14023$ & $2220 / 3987$ & $594 / 1101$ \\
\hline \multirow[t]{2}{*}{ ENF 16S 191} & \multirow[t]{2}{*}{ Enterococcus faecalis } & $47.5 \%$ & $18.8 \%$ & $0 \%$ \\
\hline & & $322 / 678$ & $3 / 16$ & $0 / 1$ \\
\hline ENU 23S 140 Beimfohr & Enterococcus faecium & $36 / 37$ & & $1 / 4$ \\
\hline \multirow[t]{2}{*}{ EGA 16S 141} & \multirow[t]{2}{*}{ Enterococcus gallinarum } & $66.7 \%$ & - & - \\
\hline & & $2 / 3$ & & \\
\hline \multirow[t]{2}{*}{ ENC 16S 221} & \multirow[t]{2}{*}{ Enterococcus spp. } & $52.4 \%$ & $11.7 \%$ & $0.3 \%$ \\
\hline & & $1403 / 2677$ & $27 / 230$ & $4 / 1260$ \\
\hline \multirow[t]{2}{*}{ ENC 176 23S kurz } & \multirow[t]{2}{*}{ Enterococcus spp. } & $86.2 \%$ & $0 \%$ & $0 \%$ \\
\hline & & $125 / 145$ & $0 / 59$ & 0/999 \\
\hline ESC $16 \mathrm{~S} 468$ & Escherichia coli & $28.3 \%$ & $29.5 \%$ & $7.9 \%$ \\
\hline Klpn23S & Klebsiella pneumoniae & $82.1 \%$ & $0 \%$ & $0 \%$ \\
\hline & & $55 / 67$ & $0 / 7$ & $0 / 2403$ \\
\hline Psae Ulm 16S 182 & Pseudomonas aeruginosa & $17.0 \%$ & $0.7 \%$ & $0.1 \%$ \\
\hline & & $747 / 4406$ & $8 / 1088$ & $6 / 4926$ \\
\hline Sal 23S 331 & Salmonella spp. & $95.1 \%$ & $75.0 \%$ & $0 \%$ \\
\hline & & $273 / 287$ & $3 / 4$ & $0 / 2236$ \\
\hline Sal Yer 23S 1705 Komp & none, prevention of cross-reaction with organisms other & $100 \%$ & $87.5 \%$ & $29.9 \%$ \\
\hline & than Salmonella spp. & $2198 / 2198$ & $239 / 273$ & $120 / 401$ \\
\hline SalYer 23S 1705 & Salmonella spp. & $94.9 \%$ & $12.5 \%$ & $0 \%$ \\
\hline & & $281 / 296$ & $34 / 271$ & $0 / 2246$ \\
\hline Komp Sal 23S 331 & none, prevention of cross-reaction with organisms other & $100 \%$ & $94.8 \%$ & $99.0 \%$ \\
\hline & than Salmonella spp. & $2181 / 2181$ & $165 / 174$ & $308 / 311$ \\
\hline Stalle $16 \mathrm{~S}$ & Staphylococcus spp. & $38.7 \%$ & $0.9 \%$ & $0.02 \%$ \\
\hline & & $2154 / 5571$ & $51 / 5484$ & $17 / 99651$ \\
\hline Stau16S Kempf & Staphylococcus aureus & $68.1 \%$ & $25.0 \%$ & $0 \%$ \\
\hline & & $451 / 662$ & $14 / 56$ & $0 / 1052$ \\
\hline Stemal Hogardt & Stenotrophomonas maltophilila & $26.9 \%$ & $14.3 \%$ & $6.7 \%$ \\
\hline & & $77 / 286$ & $315 / 2207$ & $81 / 1211$ \\
\hline Saga (variant 2: "C" at the & Streptococcus agalactiae & $65.2 \%$ & $93.9 \%$ & - \\
\hline wobble position) & & $135 / 207$ & $31 / 33$ & \\
\hline Spneu & Streptococcus pneumoniae & $97.4 \%$ & $1.6 \%$ & $0.3 \%$ \\
\hline & & $683 / 701$ & $20 / 1220$ & $7 / 2050$ \\
\hline Spn Komp & none, prevention of cross-reaction with bacteria other & $98.6 \%$ & $74.8 \%$ & $99.9 \%$ \\
\hline & than Streptococcus pneumoniae & $1197 / 1214$ & $2051 / 2741$ & $2141 / 2144$ \\
\hline Strep Franks $=$ Str $16 \mathrm{~S} 492$ Jansen & Streptococcus spp. & $43.6 \%$ & $3.1 \%$ & $0.03 \%$ \\
\hline & & $3725 / 8553$ & $39 / 1239$ & $10 / 39762$ \\
\hline Bact 16 S 389 & Bacteroides spp. & $2.2 \%$ & $0.3 \%$ & $0.2 \%$ \\
\hline & & $463 / 21395$ & $27 / 9376$ & $17 / 9436$ \\
\hline Bact Komp & none, prevention of cross-reaction with Porphyromonas spp. & $99.3 \%$ & $98.4 \%$ & $99.9 \%$ \\
\hline & & $3110 / 3132$ & $29461 / 29941$ & $16677 / 16691$ \\
\hline Bact Komp 2 & none, prevention of cross-reaction with Prevotella spp. & $100 \%$ & $97.9 \%$ & $99.5 \%$ \\
\hline & & $10857 / 10858$ & $21873 / 22351$ & $4896 / 4921$ \\
\hline Mic & Micrococcus spp. & $78.4 \%$ & $34.0 \%$ & $4.5 \%$ \\
\hline & & $355 / 453$ & $34 / 100$ & $24 / 528$ \\
\hline Spy1 & Streptococcus pyogenes & $72.2 \%$ & $100 \%$ & $0 \%$ \\
\hline & & $143 / 198$ & $1 / 1$ & $0 / 2$ \\
\hline
\end{tabular}

process is finished and better databases are available, designing better FISH probes for anaerobic bacteria may be possible in the future.

While the newly introduced Bacterioides spp. probe showed limited reliability, considerably better results were observed for the new probes for Micrococcus spp., for $S$. pyogenes, and, to a letter extent, for the probe targeting $P$. aeruginosa. Accordingly, these new probes represent useful additions to diagnostic FISH probe panels. In our hands, the newly introduced $S$. pyogenes probe was more reliable than the previously published one [26] (data not shown), and it could be useful to evaluate it with other sample materials as well.

For several published FISH probes and probe-competitorprobe combinations, acceptable performance characteristics could be shown. Accordingly, FISH can provide rapid preliminary pathogen identification from positive blood culture materials in resource-limited settings, where sophisticated and expensive approaches like MALDI-TOF-MS are not available for financial reasons. If published DNA-probes without patent protection are used, the price of material costs for a FISH reaction is less than one dollar if basic laboratory equipment like an incubator and a fluorescence microscope is available. The application of the multi-probe concept as described above reduces the risk of false positive results, making this algorithm based on Gram-morphology highly useful for FISHapplications. As usual for molecular methods, however, inhouse FISH probe panels have to be thoroughly evaluated for diagnostic purposes and cannot just be blindly taken from previous publications to be applied in the diagnostic routine setting. 
Table 5. Performance characteristics of the assessed FISH probes

\begin{tabular}{|c|c|c|c|c|}
\hline Target organism & $\begin{array}{c}\text { Sensitivity } \\
(\% ; \text { absolute numbers })\end{array}$ & $\begin{array}{c}\text { Specificity } \\
(\% ; \text { absolute numbers })\end{array}$ & $\begin{array}{l}\text { Positive predictive value } \\
\text { (\%; absolute numbers) }\end{array}$ & $\begin{array}{l}\text { Negative predicitive value } \\
(\% \text {; absolute numbers })\end{array}$ \\
\hline S. aureus & $96.4,81 / 84$ & $100.0,450 / 450$ & $100.0,81 / 81$ & $99.3,450 / 453$ \\
\hline Staphylococcus spp. & $98.3,515 / 524$ & $100.0,10 / 10$ & $100.0,515 / 515$ & $52.6,10 / 19$ \\
\hline E. faecalis & $94.7,36 / 38$ & $100.0,110 / 110$ & $100.0,36 / 36$ & $98.2,110 / 112$ \\
\hline E. faecium & $80.5,33 / 41$ & $100.0,107 / 107$ & $100.0,33 / 33$ & $93.0,107 / 115$ \\
\hline E. gallinarum & $100.0,3 / 3$ & $98.6,143 / 145$ & $60.0,3 / 5$ & $100.0,143 / 143$ \\
\hline Enterococcus spp. & $96.3,79 / 82$ & $100.0,66 / 66$ & $100.0,79 / 79$ & $95.7,66 / 69$ \\
\hline S. pneumoniae & $87.5,14 / 16$ & $100.0,132 / 132$ & $100.0,14 / 14$ & $98.5,132 / 134$ \\
\hline S. pyogenes & $100.0,3 / 3$ & $99.3,144 / 145$ & $75.0,3 / 4$ & $100.0,144 / 144$ \\
\hline Streptococcus spp. & $98.4,62 / 63$ & $96.5,82 / 85$ & $95.4,62 / 65$ & $98.8,82 / 83$ \\
\hline Acinetobacter spp. & $100.0,9 / 9$ & $100.0,243 / 243$ & $100.0,9 / 9$ & $100.0,243 / 243$ \\
\hline Bacteroides spp. & $28.6,2 / 7$ & $100.0,245 / 245$ & $100.0,2 / 2$ & $98.0,245 / 250$ \\
\hline Bacteroides spp./ & $57.1,4 / 7$ & $100.0,245 / 245$ & $100.0,4 / 4$ & $98.8,245 / 248$ \\
\hline \multicolumn{5}{|l|}{ Prevotella spp. } \\
\hline Enterobacteriaceae & $98.0,199 / 203$ & $100.0,49 / 49$ & $100.0,199 / 199$ & $92.5,49 / 53$ \\
\hline Klebsiella pneumoniae & $100.0,27 / 27$ & $98.2,221 / 225$ & $87.1,27 / 31$ & $100.0,221 / 221$ \\
\hline Pseudomonas & $90.0,27 / 30$ & $100.0,222 / 222$ & $100.0,27 / 27$ & $98.7,222 / 225$ \\
\hline \multicolumn{5}{|l|}{ aeruginosa } \\
\hline Salmonella spp. & $100.0,6 / 6$ & $100.0,246 / 246$ & $100.0,6 / 6$ & $100.0,246 / 246$ \\
\hline $\begin{array}{l}\text { Stenotrophomonas } \\
\text { maltophilia }\end{array}$ & $66.7,2 / 3$ & $100.0,249 / 249$ & $100.0,2 / 2$ & $99.6,249 / 250$ \\
\hline
\end{tabular}

Table 6. Details of lacking or incorrect binding of specific FISH probes

\begin{tabular}{|c|c|c|}
\hline Probe & Failed to identify & Cross bindings \\
\hline S. aureus & 3/84 S. aureus & \\
\hline \multirow[t]{3}{*}{ Staphylococcus spp. } & $2 / 84 S$. aureus & 1/18 S. mitis ${ }^{a}$ \\
\hline & 3/356 S. epidermidis & \\
\hline & coagulase negative staphylococci & \\
\hline E. faecalis & $2 / 38$ E. faecalis & \\
\hline E. faecium & 8/41 E. faecium & \\
\hline E. gallinarium & & 2/41 E. faecium \\
\hline Enterococcus spp. & 2/41 E. faecium & \\
\hline S. agalactiae & $1 / 5 S$. agalactiae & \\
\hline S. pneumoniae & 2/16 S. pneumoniae & \\
\hline S. pyogenes & & $1 / 6 S$. anginosus \\
\hline Streptococcus spp. & 1/18 S. mitis & 3/3 Lactococcus lactis \\
\hline \multirow{2}{*}{$\begin{array}{l}\text { Bacteroides sp. probe in conjunction with the competitor } \\
\text { probes against Prevotella spp. and Porphyromonas spp. }\end{array}$} & 3/4 Bacteroides fragilis & \\
\hline & $\begin{array}{l}\text { 1/1 Bacteroides thetaiotaomicron } \\
\text { 1/2 Bacteroides spp. }\end{array}$ & \\
\hline Bacteroides sp./Prevotella sp. probe in conjunction with & 2/2 Bacteroides spp. & \\
\hline only the competitor probe against Porphyromonas spp. & 1/4 Bacteroides fragilis & \\
\hline Enterobacteriaceae & 4/126 E. coli & \\
\hline Escherichia coli & 5/126 E. coli & 1/7 Klebsiella oxytoca \\
\hline \multirow[t]{3}{*}{ Klebsiella pneumoniae } & & 1/1 E. amnigenes \\
\hline & & 2/7 Klebsiella oxytoca \\
\hline & & 1/4 Serratia marcescens \\
\hline P. aeruginosa & 3/30 P. aeruginosa & \\
\hline S. maltophilia & $1 / 3$ S. maltophilia & \\
\hline
\end{tabular}

\section{Conclusions}

In summary, the described FISH panel is a suitable approach for rapid preliminary identification of pathogens from blood culture materials, which could be useful for resourcelimited settings. Individual misidentifications may occur. The demonstrated performance under diagnostic routine-like conditions confirms the suitability of the approach for the routine setting, apart from study conditions. However, FISH probe panels have to be thoroughly adapted to individual diagnostic needs. To our experience, FISH for the rapid identification of S. aureus was particularly useful.

\section{Funding Sources}

There has been no source of funding.

\section{Authors' Contributions}

AR performed the assessments and was mainly responsible for the publication. SP planned and conducted the experiments. MR also conducted the experiments. HF supervised and supported AR's assessments. All authors jointly wrote and corrected the manuscript.

\section{Conflict of Interest}

Nothing to report.

\section{References}

1. Frickmann H, Zautner AE, Moter A, Kikhney J, Hagen RM, Stender H, et al. Fluorescence in situ hybridization (FISH) in the microbiological diagnostic routine laboratory: a review. Crit Rev Microbiol. 2017;43:263-93. 
2. Kempf VAJ, Trebesius K, Autenrieth IB. Fluorescent in situ hybridisation allows rapid identification of microorganisms in blood cultures. J Clin Microbiol. 2000;38:830-8.

3. Gosiewski T, Kasprzyk A, Strus M. Comparision of the sensitivity of detection of bacteria in human blood using classic culture methods and molecular techniques: PCR and FISH. Med Dosw Mikrobiol. 2005;57:319-25.

4. Wellinghausen N, Bartel M, Essig A, Poppert S. Rapid identification of clinically relevant Enterococcus species by fluorescence in situ hybridization. J Clin Microbiol. 2007;45:3424-6.

5. Poppert S, Riecker M, Wellinghausen N, Frickmann H, Essig A. Accelerated identification of Staphylococcus aureus from blood cultures by a modified fluorescence in situ hybridization procedure. J Med Microbiol. 2010;59:65-8.

6. Frickmann H, Essig A, Hagen RM, Riecker M, Jerke K, Ellison D, et al. Rapid identification of Acinetobacter spp. by fluorescence in situ hybridization (FISH) from colony and blood culture material. Eu J Microbiol Imunol (Bd). 2011:1:289-96.

7. Frickmann H, Hänle A, Essig A, Dekker D, Boahen K, Acquah S, et al. Fluorescence in situ hybridization (FISH) for rapid identification of Salmonella spp. from agar and blood culture broth - An option for the tropics? In J Med Microbiol. 2013;303:277-84.

8. Clerc O, Prod'hom G, Vogne C, Bizzini A, Calandra T, Greub G. Impact of matrix-assisted laser desorption ionization time-of-flight mass spectrometry on the clinical management of patients with Gram-negative bacteremia: a prospective observational study. Clin Infect Dis. 2013:56:1101-7.

9. March-Rosselló GA, Muñoz-Moreno MF, García-Loygorri-Jordán de Urriés MC, Bratos-Pérez MA. A differential centrifugation protocol and validation criterion for enhancing mass spectrometry (MALDI-TOF) results in microbial identification using blood culture growth bottles. Eur J Clin Microbiol Infect Dis. 2013;32:699-704.

10. Søgaard M, Stender H, Schønheyder HC. Direct identification of major blood culture pathogens, including Pseudomonas aeruginosa and Escherichia coli, by a panel of fluorescence in situ hybridization assays using peptide nucleic acid probes. J Clin Microbiol. 2005;43:1947-9.

11. Søgaard M, Hansen DS, Fiandaca MJ, Stender H, Schønheyder HC. Peptide nucleic acid fluorescence in situ hybridization for rapid detection of Klebsiella pneumoniae from positive blood cultures. J Med Microbiol. 2007;56:914-7.

12. Peleg AY, Tilahun Y, Fiandaca MJ, D'Agata EMC, Venkataraman L, Moellering Jr. RC, et al. Utility of peptide nucleic acid fluorescence in situ hybridization for rapid detection of Acinetobacter spp. and Pseudomonas aeruginosa. J Clin Microbiol. 2009;47:830-2.

13. Morgan M, Marlowe E, Della-Latta P, Salimnia H, Novak-Weekley S, $\mathrm{Wu} F$, et al. Multicenter evaluation of a new shortened peptide nucleic acid fluorescence in situ hybridization procedure for species identification of select Gram-negative bacilli from blood cultures. J Clin Microbiol. 2010;48:2268-70.

14. Della-Latta P, Salimnia H, Painter T, Wu F, Procop GW, Wilson DA, et al. Identification of Escherichia coli, Klebsiella pneumoniae, and Pseudomonas aeruginosa in blood cultures: a multicenter performance evaluation of a three-color peptide nucleic acid fluorescence in situ hybridization assay. J Clin Microbiol. 2011;49:2259-61.

15. Leitner E, Scherr S, Strempfl C, Krause R, Feierl G, Grisold AJ. Rapid Identification of Pathogens with the hemoFISH Test Applying a Nove
Beacon-Based Fluorescence in Situ Hybridization (bbFISH) Technology in Positive Blood Culture Bottles. J Mol Diagn. 2013;15:835-9.

16. Poppert S, Riecker M, Essig A. Rapid identification of Propionibacterium acnes from blood cultures by fluorescence in situ hybridization. Diagn Microbiol Infect Dis. 2010;66:214-6.

17. Peters RP, Savelkoul PH, Simoons-Smit AM, Danner SA, Vandenbroucke-Grauls CM, van Agtmael MA. Faster identification of pathogens in positive blood cultures by fluorescence in situ hybridization in routine practice. J Clin Microbiol. 2006;44:119-23.

18. Wong EH, Subramaniam G, Navaratnam P, Sekaran SD. Rapid detection of non-Enterobacteriaceae directly from positive blood culture using fluorescent in situ hybridization. Indian J Med Microbiol. 2007;25:391-4.

19. Gescher DM, Kovacevic D, Schmiedel D, Siemoneit S, Mallmann C, Halle E, et al. Fluorescence in situ hybridisation (FISH) accelerates identification of Gram-positive cocci in positive blood cultures. Int $\mathrm{J}$ Antimicrob Agents. 2008;32:S51-9.

20. Horváth A, Kristóf K, Konkoly-Thege M, Nagy K. Rapid identification of pathogens in blood culture with fluorescent in situ hybridization (FISH). Acta Microbiol Immunol Hung. 2010;57:225-34

21. Sönksen UW, Christensen JJ, Nielsen L, Hesselbjerg A, Hansen DS, Bruun B. Fastidious Gram-Negatives: Identification by the Vitek 2 NeisseriaHaemophilus Card and by Partial 16S rRNA Gene Sequencing Analysis. Open Microbiol J. 2010;4:123-31.

22. Kierzkowska M, Majewska A, Kuthan RT, Sawicka-Grzelak A, Młynarczyk G. A comparison of Api 20A vs MALDI-TOF MS for routine identification of clinically significant anaerobic bacterial strains to the species level. J Microbiol Methods. 2013;92:209-12.

23. Carretto E, Bardaro M, Russello G, Mirra M, Zuelli C, Barbarini D. Comparison of the Staphylococcus QuickFISH BC test with the tube coagulase test performed on positive blood cultures for evaluation and application in a clinical routine setting. J Clin Microbiol. 2013;51:131-5.

24. Lakner A, Essig A, Frickmann H, Poppert S. Evaluation of fluorescence in situ hybridisation (FISH) for the identification of Candida albicans in comparison with three phenotypic methods. Mycoses. 2012;55: e114-23.

25. Frickmann H, Lakner A, Essig A, Poppert S. Rapid identification of yeast by fluorescence in situ hybridisation from broth and blood cultures. Mycoses. 2012;55:521-31.

26. Hogardt M, Trebesius K, Geiger AM, Hornef M, Rosenecker J, Heesemann J. Specific and rapid detection by fluorescent in situ hybridization of bacteria in clinical samples obtained from cystic fibrosis patients. J Clinical Microbiol. 2000;38:818-25.

27. Amann RI, Krumholz L, Stahl DA. Fluorescent-oligonucleotide probing of whole cells for determinative, phylogenetic, and environmental studies in microbiology. J Bacteriol. 1990;172:762-70.

28. Trebesius K, Leitritz L, Adler K, Schubert S, Autenrieth IB, Heesemann J. Culture independent and rapid identification of bacterial pathogens in necrotizing fasciitis and streptococcal toxic shock syndrome by fluorescence in situ hybridization. Med Microbiol Immunol. 2000;188:169-75.

29. Franks AH, Harmsen HJ, Raangs GC, Jansen GJ, Schut F, Welling GW. Variations of bacterial populations in human feces measured by fluorescent in situ hybridization with group-specific 16S rRNA-targeted oligonucleotide probes. Appl Environ Microbiol. 1998;64:3336-45. 